\title{
1) Perspectivas del conocimiento especializado del profesor de matemáticas como elemento de su desarrollo profesional
}

\section{- Perspectives of the Specialized Knowledge of Math Teachers as an Element of Their Professional Development}

- Perspectivas do conhecimento especializado do professor de matemáticas como elemento do seu desenvolvimento profissional

\section{Resumen}

Este artículo de investigación presentan los aportes teóricos al conocimiento especializado del profesor de matemáticas que se han tenido como elementos bases del desarrollo profesional planteados en la elaboración de la Tesis de Doctorado titulada "Desarrollo profesional del profesor de matemáticas: estudio de caso en el nivel medio de secundaria". En un primer momento se retoman las definiciones de conocimiento del profesor y conocimiento profesional de Shulman (1986) y Ball, Thames y Phelps (2008) y se justifica el paso a la propuesta del conocimiento especializado de las investigaciones de Muñoz-Catalán y otros (2015). Seguido a lo anterior, se aborda el concepto de desarrollo profesional del profesor y por último se propone una tercera estructura del Conocimiento Especializado del Profesor de Matemáticas con tres componentes: conformación de guiones, conformación de imágenes y conformación de técnicas concretas, Se concluye que el conocimiento de la experiencia junto con la reflexión permiten que el profesor se desarrolle profesionalmente.

\section{Palabras clave}

Desarrollo profesional; conocimiento especializado; profesor de matemáticas educación matemática

\footnotetext{
Abstract

This research paper presents the theoretical contributions to the specialized knowledge of the math teacher that have been used as basic elements for professional development in the elaboration of Doctorate Thesis titled "Professional development of the math teacher: case study at the middle school level." On a first instance, the definitions of teacher knowledge and professional knowledge by Shulman (1986) and Ball, Thames and Phelps (2008) are revisited, and the change to the proposal of specialized knowledge of research by Muñoz-Catalán et al. (2015) is explained. Accordingly, the concept of professional development of the teacher is addressed and, finally, a third structure of Specialized Knowledge
}

Isaac Lima-Díaz*

Magíster en Enseñanza de las Ciencias Experimentales y Matemáticas. Candidato a Doctor en Ciencias de la Educación, Universidad Nacional de La Plata. Profesor del Departamento de Matemáticas, Universidad Pedagógica Nacional. Correo electrónico: ilimad@pedagogica.edu.co Orcid: 0000-0002-5045-3409 
of the Math Teacher with three components is proposed: creation of scripts; creation of images; and creation of specific techniques. It is concluded that the knowledge of experience and reflection enable the teacher's professional development.

Keywords

Professional development; specialized knowledge; math teacher; math education

\section{Resumo}

Este artigo de pesquisa apresenta as contribuições teóricas ao conhecimento especializado professor de matemática, consideradas elementos base do desenvolvimento profissional na tese de Doutoramento intitulada "Desenvolvimento profissional do professor de matemáticas: estudo de caso na educação média". Primeiramente, são retomadas as definições de conhecimento do professor e conhecimento profissional de Shulman (1986) e Ball, Thames e Phelps (2008) e justifica-se a proposta do conhecimento especializado das pesquisas de Muñoz-Catalán e outros (2015). Em seguida, aborda-se o conceito de desenvolvimento profissional do professor e, por último, propõe-se uma terceira estrutura do conhecimento especializado do professor de matemáticas com três componentes: construção de roteiros, construção de imagens, e construção de técnicas concretas. Conclui-se que o conhecimento da experiência junto com a reflexão permitem o desenvolvimento profissional do professor.

Palavras-chave

Desenvolvimento profissional; conhecimento especializado; professor de matemática; educação matemática 


\section{Introducción}

Las investigaciones sobre la práctica del profesor han considerado a lo largo de varias décadas el conocimiento que este debe tener al momento de su actuación como profesional. En este sentido, se han identificado terminologías para referirse al mismo: conocimiento del profesor, conocimiento práctico del profesor, conocimiento profesional del profesor, conocimiento especializado, aunque el fondo de sus marcos teóricos pueda diferir entre sí.

El estudio y el análisis del conocimiento que necesita el profesor supone una profundización en y desde la matemática, por lo que requiere profundizar en los objetos matemáticos implicados, sus raíces epistemológicas, los fenómenos de los que emergen, sus relaciones con otros objetos matemáticos, la estructura que estas relaciones permiten construir o la forma en que estos entes matemáticos se construyen. Referirse a dicho conocimiento como conocimiento del profesor puede ser considerado como un elemento amplio: el profesor tiene otros conocimientos que no necesariamente involucra con su actividad docente, incluso pueden ser contradictorios y no aportar al ejercicio de su profesión.

El término conocimiento práctico lo puede limitar a su actividad en el aula de clase obviando elementos que en el desarrollo de la actividad no necesariamente provienen de lo práctico, tal como la teoría o las propias concepciones. El término conocimiento profesional no permite colegir una significación como la que se pretende asignar (Guacaneme, 2013), dado las acepciones que tienen los términos profesional y profesión, aunque se reconoce la intención de llevar al entendimiento de la actividad del profesor como una profesión que va más allá de la condición de oficio, labor técnica o tecnológica, arte, concibiendo al profesor como un profesional y no como un operario, técnico, artista o artesano. Así, el conocimiento requerido para desempeñarse en la profesión docente compila (Eisenberg, 1977) saberes académicos que provienen de diferentes disciplinas que se alimentan por medio de la investigación, se retroalimenta de la reflexión y se condiciona a partir de especificidades sociales, políticas y culturales.

En este sentido, el análisis de la práctica del profesor no se puede realizar sin una referencia a un conocimiento matemático (abordado a partir del contexto específico en el que se emplea) y a los procesos de enseñanza y de aprendizaje de dicho conocimiento, otorgando a dicho conocimiento matemático de un carácter especializado y, por tanto, específico de la labor del profesor de matemáticas. De manera análoga que las matemáticas están constituidas por diversas ramas o especializaciones, el conocimiento matemático del profesor y la tarea que supone su identificación y análisis pertenecerían a otro tipo de aplicación de las matemáticas en los ámbitos educativos. El análisis del conocimiento que necesita el profesor supone una profundización en y desde las matemáticas y las matemáticas escolares, por lo que requiere profundizar en los objetos matemáticos implicados, sus raíces epistemológicas, los fenómenos de los que emergen, sus relaciones con otros objetos matemáticos, la estructura que estas relaciones permiten construir o la forma en que estos entes matemáticos se construyen, los errores y los obstáculos en su enseñanza y aprendizaje.

El enfoque del conocimiento especializado del profesor de matemáticas se centra en la aplicación específica en los procesos de enseñanza y aprendizaje, es decir, en cada momento en que interaccionan las matemáticas, los alumnos y el profesor. Ciertamente, otros factores influyen en esta interacción, como la cultura del centro, su entorno social, las normas del aula, entre otros. No obstante, interesan 
primordialmente el conocimiento del profesor sobre los temas que ha de enseñar, sobre las relaciones entre objetos matemáticos o sobre la forma de hacer matemáticas, así como sobre estrategias y recursos para enseñar matemáticas, sobre las dificultades de aprendizaje de los alumnos o sus errores habituales, y sobre lo que puede esperarse que aprendan los alumnos en un nivel determinado.

Las diferentes investigaciones sobre el conocimiento especializado del profesor han mostrado que poseer un conocimiento adecuado del contenido no siempre se corresponde con la adecuada capacidad para enseñarlo (Eisenberg, 1977), (Begle, 1979), (Askew, Brown, Rhodes, Johnson y Wiliam, 1997), pero sí una buena disposición para lograr dicha habilidad (Hill, Rowan y Ball, 2005). Esto significa que, además de requerimientos de carácter psicológicos y pedagógicos, cuya utilidad no son el foco principal de este documento, los profesores adquieren un conocimiento de naturaleza diferente del contenido a enseñar. Ball, Thames y Phelps (2008) realizaron una aportación relevante en este sentido, considerando dentro del dominio del conocimiento matemático un subdominio que se denominará Conocimiento Especializado del Contenido, diferente al Conocimiento Común que cualquier persona matemáticamente instruida puede poseer. El equipo de Ball (2000) comenta que el Conocimiento Común hace parte de la cultura general de quien ha aprendido matemáticas, a diferencia del Conocimiento Especializado del Contenido, que se refiere no solamente a dicha cultura general, sino que abarca otras situaciones como, por ejemplo, conocer diferentes representaciones de un concepto matemático, las relaciones con matemáticas aplicadas y el tratamiento con la práctica matemática.

\section{Conocimiento del profesor, conocimiento didáctico del contenido, conocimiento matemático para la enseñanza}

Al identificar y describir el conocimiento del profesor, parece necesario diferenciar elementos que ayuden a precisar el contenido, además de la organización y la estructura del mismo. Los elementos se pueden identificar como componentes del conocimiento, aunque algunos autores para evitar confusiones se refieren a ellos como tipos - del conocimiento dominante- y fuentes - del conocimiento deseable- (Porlán y Rivero, 1998).

A lo largo del tiempo, se han considerado algunos términos para referirse al conocimiento que debe tener el profesor de matemáticas integrando el conocimiento disciplinar y el didáctico, diferenciando el conocimiento de las matemáticas y el conocimiento didáctico del contenido. Shulman (1986) plantea que se debe enfocar el conocimiento necesario para enseñar la disciplina específica a través del concepto conocimiento didáctico del contenido comprendiéndolo como un tipo de conocimiento del contenido que incorpora los aspectos del contenido más relacionados con la enseñanza; no obstante, este tipo de conocimiento no se puede identificar fácilmente en la práctica. 
Como consecuencia de lo anterior, Ball, Thames y Phelps (2008) proponen organizar y operativizar el conocimiento del profesor de matemáticas por medio de investigaciones sobre la práctica, las cuales compilan en lo que denominan conocimiento matemático para la enseñanza, representando el conocimiento matemático, habilidades, hábitos de la mente (estrategias para cálculos, uso de propiedades de manera instantánea, estrategias para la solución de situaciones, entre otros), sensibilidad y otros elementos requeridos en el trabajo concreto de enseñar y en las tareas especializadas en las que los profesores necesitan conocer y usar las matemáticas.

En este modelo, el conocimiento de las matemáticas está compuesto por el conocimiento común del contenido referido al que se supone cualquier adulto culto posee (Ball, Thames y Phelps, 2008), el conocimiento especializado del contenido que tiene sentido para el profesor de matemáticas y el conocimiento en el horizonte matemático que permite interrelacionar contendidos a lo largo del currículo y valorar y reconocer la validez de los razonamientos y resoluciones de los estudiantes'. El conocimiento especializado de contenido es un contenido estrictamente matemático que el profesor necesita y usa que no es necesariamente requerido por otros profesionales con formación matemática (Ball, Thames y Phelps, 2008; Bass, Didis, Erbas, Cetinkaya, Cakiroglu y Alacaci, 2013), ni tampoco es un subconjunto de lo que los matemáticos conocen.
El conocimiento didáctico del contenido está compuesto por el conocimiento del contenido y de cómo los estudiantes se apropian de él. Permite comprender el contenido desde la perspectiva del aprendizaje y la enseñanza e implica argumentar por qué un determinado material, recurso o representación es apropiada para explicar un determinado concepto.

\section{Conocimiento especializado del profesor de matemáticas}

El modelo del conocimiento matemático para la enseñanza aporta elementos a la conceptualización del conocimiento del profesor: redefine las ideas de las investigaciones de Shulman sobre el conocimiento didáctico del contenido, la idea del conocimiento del profesor como exclusivo y la visión transversal de las matemáticas escolares (Shulman y Grossman, 1988; Shulman y Simon, 1992; Shulman, 1987). Asimismo, permite discutir sobre cuatro elementos: el conocimiento en el horizonte matemático, la manera en que se especializa el conocimiento del contenido, posible solapamiento de subdominios entre el conocimiento del contenido y el conocimiento didáctico del contenido y la falta de matematización del modelo.

La siguiente gráfica presenta la variación acerca de la idea acerca del conocimiento del profesor que se detalló en los párrafos anteriores:

\footnotetext{
Ball, Thames y Phelps (2008) establecen que el Conocimiento del Horizonte Matemático es un tipo de conocimiento que se refiere a la conciencia del profesor sobre los conocimientos matemáticos previos y futuros presentes en el currículo. Esto implica que el profesor adquiera una visión global de la educación matemática de los estudiantes para ser empleada al enseñar matemáticas en el aula.
} 
Conocimiento didáctico del

contenido

- Aspectos relacionados con la enseñanza de las matemáticas

- Aspectos relacionados

con el aprendizaje de las matemáticas
Conocimiento matemático

para la enseñanza

- Conocimiento común del contenido

- Conocimiento especializado del contenido

- Conocimiento del horizonte matemático
Conocimiento especializado del profesor de Matemáticas

- Conocimiento matemático:

Temas, Estructura Matemática y Práctica Matemática

- Conocimiento didáctico

de contenido matemático: Enseñanza, Características de aprendizaje y Estándares

Figura 1. Modificaciones sobre las teorías acerca del conocimiento de profesor Fuente: elaboración propia

Respecto del conocimiento en el horizonte matemático, Ball y Bass (2009) afirman que este incluye el conocimiento de ideas y estructuras de las matemáticas y las conexiones entre diferentes entes tales como conceptos y propiedades. Dichas conexiones se manifiestan de dos maneras: intraconceptuales e interconceptuales. Las conexiones intraconceptuales establecen la proximidad con un concepto; determinan la equivalencia entre caracterizaciones o prueban la equivalencia entre dos definiciones, distinguiendo entre una condición suficiente de una necesaria. Las conexiones interconceptuales ocurren cuando los conectores son tratados como ideas matemáticas que permiten vincular diferentes representaciones del mismo concepto o diferentes conceptos que los estudiantes afrontan en el mismo momento; o temporales, cuando se dan entre conocimientos previos y futuros. El conocimiento de las ideas y estructuras matemáticas y las conexiones entre entes influyen en el conocimiento del profesor sobre los conocimientos previos y futuros de los estudiantes. Estas conexiones posibilitan estudiar otras propiedades de un concepto o procedimiento o aplicar el conocimiento aprendido a situaciones nuevas o más complejas.

El conocimiento del horizonte matemático también vincula conocimiento de tipo sintáctico (Schwab, 1978), aspectos de la comunicación matemática, razonamiento y prueba, definiciones, relaciones, correspondencias y equivalencias. Resalta los valores de las matemáticas como el gusto por la coherencia de los argumentos y la precisión y cuidado en la consistencia del lenguaje matemático.

Acerca de la manera en la que se especializa el conocimiento de contenido, Ball y Bass (2009) caracterizan el conocimiento común del contenido como aquel que puede poseer una persona instruida. En este sentido, al momento de decidir si un episodio de información, entendido como el momento de la observación que brinda información sobre el conocimiento de horizonte matemático, aporta evidencias sobre conocimiento común del contenido, se debe comparar con un 
conocimiento hipotético de alguien que pueda tener ciertos conocimientos deseables, obligando a establecer referentes y discusiones de lo que es o no común.

El conocimiento de contenido y el conocimiento didáctico del contenido se pueden solapar cuando el profesor necesita conocer de dónde vienen los errores y los canales en los que se lleva a cabo el razonamiento de los estudiantes. Este estudio es exclusivo del profesor de matemáticas y no se asocia con otras profesiones; sin embargo, el estudio de las dificultades y el momento en el que se cometen errores se asocia con el conocimiento del contenido y los estudiantes, a diferencia del estudio de la procedencia matemática del error, la cual está situada en el conocimiento especializado del contenido.

El modelo de Ball (1990) es un refinamiento de las teorías de Shulman (1986) y no ha sido matematizado. A excepción del conocimiento en el horizonte matemático, los subdominios del conocimiento matemático para la enseñanza no son explícitos al contenido matemático o a las matemáticas aplicadas, comprendiendo su aplicación a la enseñanza.

Con base en esos cuatro puntos de discusión, Muñoz-Catalán et al. (2015) consideran que la especialización del profesor deriva de su profesión (profesor de matemáticas) en la que el ejercicio de la enseñanza es un elemento definitorio, y que el modelo de enseñanza de las matemáticas debe estar conceptualizado en el mismo hecho que (este modelo) se usa para comprender el conocimiento que el profesor de matemáticas usa para enseñar matemáticas. El conocimiento del profesor, en la forma como lo que sabe y sabe hacer respecto a la enseñanza de las matemáticas, es una red de conocimientos y destrezas con diferentes niveles de asentamiento, integración, accesibilidad o activación en la práctica.

\section{Componentes del conocimiento especializado del profesor}

El conocimiento especializado del profesor de matemáticas se concreta, por medio de sus fuentes, en seis componentes, de los cuales tres son para el conocimiento matemático: conocimiento de los temas, de la estructura de la matemática y la práctica matemática. Los otros tres están dirigidos al conocimiento didáctico del contenido: conocimiento de la enseñanza de las matemáticas, de las características del aprendizaje de las matemáticas y de los estándares de aprendizaje de las matemáticas.

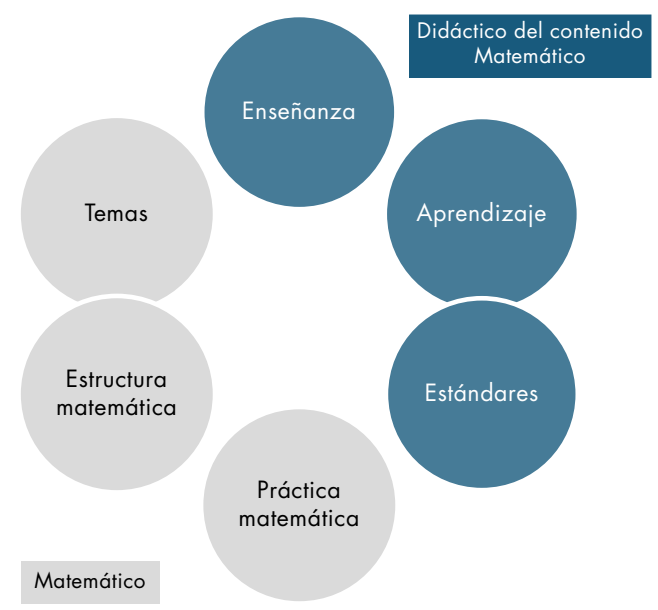


A continuación, se procede a describir cada uno de esos componentes.

\section{Conocimiento de los temas}

Es un conocimiento profundo del contenido escolar (se entiende que el profesor debe saber contenido más allá de lo que los estudiantes aprenden) e incluye aspectos de los conceptos que les permiten relacionarlos con contextos reales y aportar elementos epistemológicos ligados a las matemáticas que llevan a la comprensión de diferentes significados que pueden atribuirse al contenido y los contextos en los que se sitúan. Este conocimiento no se limita al contenido objeto de enseñanza y de aprendizaje, va más allá del conocimiento de las matemáticas como disciplina, las matemáticas escolares, los fundamentos matemáticos, los procedimientos, estándares y formas de la representación de los diferentes temas.

\section{Conocimiento de la estructura matemática}

En coherencia con el ítem anterior, que contempla un conocimiento más amplio de las matemáticas que está enseñando, el conocimiento de la estructura matemática abarca el conocimiento de las matemáticas desde la integración y la relación en estructuras amplias que pueden ir de lo local a lo universal. Este componente integra relaciones con conceptos avanzados y conceptos elementales, permitiendo que el profesor comprenda las matemáticas escolares desde un punto de vista superior no solo en el contenido sino en la percepción de su organización (Muñoz-Catalán et al., 2015). Este conocimiento se puede interpretar como un sistema integrado de conexiones que le permita comprender y desarrollar conceptos avanzados desde perspectivas elementales y conceptos elementales mediante el tratamiento de visiones avanzadas.

\section{Conocimiento de la práctica matemática}

Se orienta directamente con la forma de hacer y ejecutar en matemáticas que debe conocer el profesor de matemáticas para desarrollar la clase: formas de mostrar y demostrar, criterios para establecer generalizaciones válidas, significado de definición, axioma y teorema como elementos constituyentes de las matemáticas, el conocimiento de la sintaxis matemática. Este componente es fundamental en el conocimiento de heurísticos en resolución de problemas que abarcan la estructura lógica en la que se desarrolla. En particular, se refiere a las formas de conocer, crear o producir en matemáticas, conocimiento de aspectos de la comunicación matemática, del razonamiento y la prueba. Saber, por ejemplo, qué es definir y cómo usar definiciones

\section{Conocimiento de la enseñanza de las matemáticas}

Este componente está referido al conocimiento que tiene el profesor de las vías, re- 
de diferentes estrategias y teorías, ya sean institucionales o personales, de la enseñanza de las matemáticas. También toma relevancia el conocimiento de diferentes recursos para la enseñanza de las matemáticas en relación con el potencial y limitaciones para la enseñanza de contenidos. No está referido a un conocimiento superficial del recurso, sino al conocimiento que se vincula con la naturaleza de los conceptos matemáticos entre sí.

\section{Conocimiento de las características del aprendizaje de las matemáticas}

Refleja el conocimiento que el profesor posee y desarrolla acerca de cómo se aprenden y piensan los contenidos matemáticos y las formas en que los estudiantes interactúan con cada contenido. Consiste en conocer las expectativas de aprendizaje que figuran en el currículo, o las que se hacen constar en la literatura de investigación, asociadas al nivel educativo en el que se aborda este aprendizaje. Un enunciado propio de este subdominio podría ser cómo en el currículo nacional se incluyen consideraciones relacionadas con las matemáticas en general y con cada contenido en particular, encontrando el conocimiento de teorías personales e institucionales, de aprendizaje, el conocimiento de fortalezas, dificultades, obstáculos o errores asociados a cada contenido, el lenguaje que los estudiantes pueden usar en cada contenido, las ideas intuitivas que ellos desarrollan en cada concepto y las actitudes hacia las matemáticas.

\section{Conocimiento de los estándares de aprendizaje de las matemáticas}

Desarrolla una visión de la noción del conocimiento curricular que abarca los grados de profundidad en que el profesor puede conocer el currículo oficial visto como el referente estandarizado (Muñoz-Catalán et al., 2015) de los contenidos y capacidades que debe aprenderse y desarrollarse en un curso etapa y las indicaciones de la forma en que deben impartirse y aprenderse los contenidos. Dichos referentes pueden estar fuera del currículo, como las propuestas de asociaciones profesionales y la opinión de los profesores expertos en el conocimiento de la práctica docente sobre qué, cómo y cuándo explicar los contenidos matemáticos.

A partir de las seis componentes anteriores y de los referentes teóricos presentados, el conocimiento especializado del profesor se puede entender como el conjunto de saberes que se adquiere en la formación inicial sobre matemática y su enseñanza, la experiencia en el aula y los saberes que de ella se desprenden, y los conocimientos teóricos que en la reflexión sobre la práctica generan el nuevo estatus de saber que se está caracterizando.

\section{El desarrollo profesional del profesor de matemáticas}

Habitualmente, en la literatura sobre el desarrollo profesional se hace referencia a los procesos de cambio, mejora o crecimiento del docente, considerado un profesional de la enseñanza, concretándolos en aspectos como el conocimiento, las concepciones y la práctica. Climent (2005) agrupa estos estudios en tres bloques diferenciados:

- Estudios que consideran que el desarrollo profesional viene definido por el acercamiento a un modelo determinado: puede ser un modelo de profesor, habitual en los estudios que consideran que el profesor es aquel cuyas características coinciden con el modelo investigativo de las tendencias didácticas presentadas en el estudio de las concepciones de esta investigación, una perspectiva curricular específica o una perspectiva consistente con 
una visión constructivista del aprendizaje (Porlán y Rivero, 1998; Schifter y Simon, 1992).

- Estudios que consideran el desarrollo profesional de manera integrada a partir de las concepciones, conocimiento y práctica: del segundo grupo de estudios, Cooney (1998) considera que el referente del desarrollo profesional es la práctica reflexiva en la que el profesor adapta su actuación al contexto. Así, el desarrollo profesional se considera como un continuo en el que se adquiere la habilidad para monitorear las acciones de acuerdo con las circunstancias en las que el profesor enseña. La enseñanza es vista como ejercicio adaptativo coherente con la visión de las matemáticas como creación humana. El contexto es una parte fundamental de la conceptualización del desarrollo, ya que tiene en cuenta aspectos de cómo una actividad puede ser o no efectiva.

- Estudios para explicar el conocimiento especializado del profesor de matemáticas: consideran que este viene determinado por el aprendizaje tanto del propio contenido matemático como del conocimiento didáctico del contenido (Muñoz-Catalán et al., 2015).

Se ha considerado una visión del desarrollo profesional que combina estos grupos. La comprensión de la práctica es nodal en el quehacer profesional del profesor: genera teoría acerca de lo que sucede en la práctica; esa comprensión se realiza en un marco ideológico, praxeológico, axiológico particular, enmarcada en un contexto que permite comprender por qué una determinada actuación es efectiva en unas determinadas situaciones y no en otras. De esta manera, el conocimiento especializado no es concebido solo como herramienta para comprender, sino como producto de esa comprensión. La reflexión se convierte tanto en el medio a través del cual se promueve el desarrollo profesional como el referente para identificar el desarrollo logrado (Climent, 2005; Llinares, 2006), a través de tres acciones que se identifican como conocimiento para, en y de la práctica (tabla 1).

Tabla 1. Comparación de las tres concepciones sobre el desarrollo profesional de Climent (2005)

\begin{tabular}{|c|c|c|c|}
\hline & $\begin{array}{l}\text { Conocimiento para } \\
\text { la práctica }\end{array}$ & Conocimiento en la práctica & Conocimiento de la práctica \\
\hline Cualidad & $\begin{array}{l}\text { Necesario para llevar } \\
\text { a la práctica }\end{array}$ & Surge en la acción & $\begin{array}{l}\text { Producto de la reflexión sobre } \\
\text { lo actuado }\end{array}$ \\
\hline Naturaleza & $\begin{array}{l}\text { Conocimiento formal, } \\
\text { proposicional, } \\
\text { declarativo, de } \\
\text { naturaleza teórica. }\end{array}$ & $\begin{array}{l}\text { Conocimiento práctico: se } \\
\text { adquiere, crece y manifiesta } \\
\text { en la acción. Inmerso en la } \\
\text { praxis y en las reflexiones de los } \\
\text { profesores sobre la misma. }\end{array}$ & $\begin{array}{l}\text { Conocimiento que emerge } \\
\text { dialécticamente entre teoría y } \\
\text { práctica. Indisociable del sujeto } \\
\text { que conoce, del contexto donde } \\
\text { lo construye y surgido a partir } \\
\text { de indagaciones intencionales. }\end{array}$ \\
\hline
\end{tabular}

Fuente: elaboración propia 
Gráficamente, el ciclo del desarrollo profesional puede ser presentado como sigue, tomando en cuenta que las líneas de puntos suponen cierre y apertura en forma simultánea (protección y permeabilidad) y que el aula, por ende la práctica, se constituye en el primer nodo del cual partir:

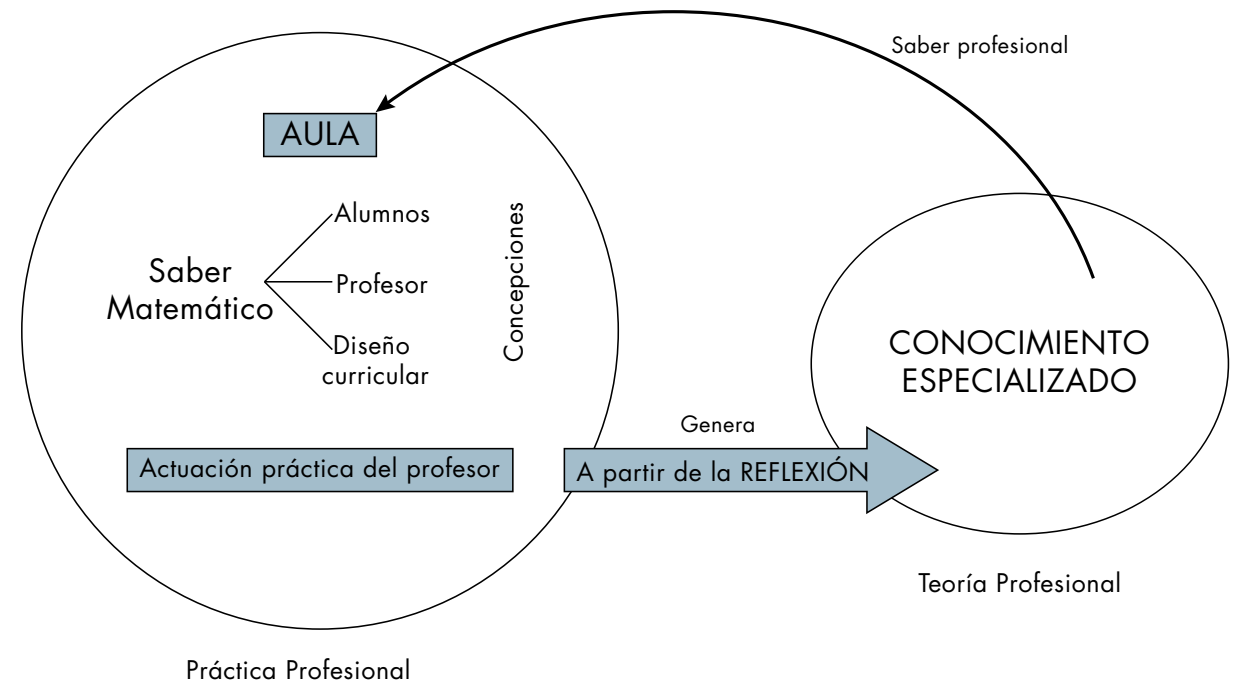

Figura 3. Desarrollo profesional del profesor de matemáticas

Fuente: elaboración propia

El desarrollo profesional puede analizarse a través de los siguientes indicadores:

Comprensión de la práctica: permite la comprensión por parte del profesor del modo de pensar propio acerca de la enseñanza, de cómo aprenden los alumnos; de modos de hablar e interactuar, observar y describir elementos del entorno y la disposición hacia la acción y la interpretación, entre otros.

Descripción de concepciones: permite entender si los errores y obstáculos de los alumnos son considerados situaciones de aprendizaje tanto para el alumno como para el profesor en lugar de ser visto como carencias de los estudiantes; si existen alternativas en los procesos de enseñanza, etc.

Conocimiento de casos: permite replantear los argumentos propios y evaluar la forma de proponerlos tomando conciencia de las concepciones y aspectos que caracterizan la práctica propia.
Problematización de la práctica: permite definir la práctica como un conjunto de problemas en las que hay muchas maneras de actuar y muchos objetivos que plantear. Esto lleva a la toma de conciencia de la complejidad de la misma y el compromiso hacia ella, siempre con actitudes de cuestionamiento y aprendizaje continuo.

Cambios en las prácticas: permiten al profesor desarrollar una metodología investigativa para determinar qué sucede en el aula cuando se implementan estrategias de enseñanza.

\section{Conocimiento de la experiencia}

El modelo del conocimiento especializado del profesor de matemáticas y su relación con el desarrollo profesional aporta elementos significativos que amplían la conceptualización del conocimiento del profesor de Shulman (1986) y de Ball (Ball, Thames y Phelps, 2008). El conocimiento matemático y cada una de sus 
componentes se refieren al saber que en matemáticas debería tener un profesor. El conocimiento de temas implica al docente en el saber de las matemáticas y las formas de representación, reconociendo que este conocimiento no se limita únicamente al que va a enseñar y supone la reflexión sobre los aspectos fenomenológicos que los involucran. Ese conocimiento matemático debe ser más amplio y no se puede limitar al de las matemáticas escolares, debe involucrar las estructuras y relaciones con un saber más puro, el cual está incluido en el conocimiento de la estructura matemática y constituye las diferentes conexiones epistemológicas entre los conceptos. Ese conocimiento de temas como de estructura matemática se establece cuando el profesor desarrolla el conocimiento de la práctica matemática que incluye las formas de hacer y proceder en matemáticas que debe conocer para desarrollar la clase y para involucrarse en los heurísticos de la resolución de problemas que abarcan la estructura lógica de esos conocimientos.

En los trabajos de grado que se tomaron como referencia para esta investigación y en el trabajo de campo realizado se observa que, a medida que el profesor adquiere ese conocimiento, va adquiriendo una serie de esquemas que se relacionan con su quehacer en matemáticas, los cuales no solamente tienen que ver de manera directa con esos conocimientos, sino también con sus concepciones, ayudando al profesor a determinar pautas de actuación frente al conocimiento matemático en sí. Dichos esquemas se van adquiriendo a medida que experimenta y profundiza en el conocimiento matemático.

Por el lado del conocimiento didáctico de contenido, el conocimiento de la enseñanza de las matemáticas, entendido como aquel acerca de diferentes estrategias y teorías tanto institucionales como personales de la enseñanza de las matemáticas, en particular herramientas e instrumentos que potencialicen el aprendizaje conectados con la naturaleza de los objetos de estudio; el conocimiento de las características del aprendizaje de las matemáticas que refleja el conocimiento que el profesor tiene acerca de cómo se aprenden los contenidos matemáticos, las formas que los estudiantes interactúan con cada contenido que incluye consideraciones específicas; no obstante, esas consideraciones están vinculadas directamente con el conocimiento de los estándares de aprendizaje de las matemáticas que relaciona los grados de profundidad con los cuales el profesor conoce el currículo oficial y los elementos de reflexión sobre la naturaleza de dicho currículo y las necesidades del contexto.

Sin embargo, poseer conocimiento acerca de la enseñanza, de las características del aprendizaje y del conocimiento de los estándares de aprendizaje de las matemáticas puede entenderse solo desde el punto de vista teórico, limitándose únicamente a ella. Se requiere que el profesor vincule ese conocimiento con su experiencia para que pueda comprender mejor ese conocimiento y para que, a medida que reflexiona, enriquezca ese conocimiento didáctico del contenido. A través de la experiencia, el profesor recolecta un tipo de conocimiento que le va a permitir enriquecer sus propias concepciones y las dos componentes primarias de su conocimiento especializado. Además del conocimiento matemático y del 
conocimiento didáctico del contenido matemático, el profesor, a medida que reflexiona desde, en y sobre la práctica va a adquirir un conocimiento de su experiencia.

Junto con los referentes teóricos, en la observación se puede determinar que, en el ejercicio de su práctica, el profesor establece guiones y esquemas de acción, imprescindibles para organizar y dirigir el curso de sus acciones como profesional; metáforas e imágenes con las cuales no cuenta en su sistema inicial de concepciones en las diferentes variables profesión y que le permiten generalizar de manera más concreta y sistematiza ideas, hipótesis de trabajo y técnicas concretas que pone de manifiesto en el posterior diseño, aplicación y seguimiento del currículo. Es por ello que se hace necesario agruparlas en una nueva componente del conocimiento especializado y denominarlas como conocimiento de la experiencia ${ }^{2}$.

A continuación, se propone la caracterización de cada una de esas componentes:

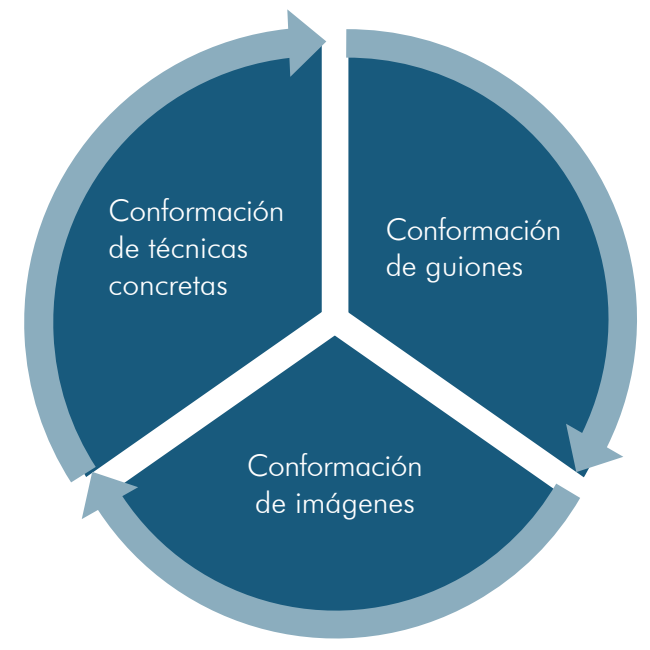

Figura 4. Componentes del conocimiento de la experiencia

Fuente: elaboración propia

2 Se hace necesario entender que, para validar este tipo de conocimiento, la experiencia no será entendida únicamente como la acumulación de tiempo a través del quehacer profesional, va ligada a los diferentes procesos de reflexión que él mismo hace a lo largo de su trayectoria.

\section{Conformación de guiones}

Referidos al conjunto de esquemas que predicen los acontecimientos del quehacer profesional del profesor de matemáticas, contienen pautas de actuación que se concretan en la asimilación de las prácticas y generan esquemas mentales simplificados de actuaciones incorporadas. En la conformación de los guiones se incluyen las rutinas que establece el profesor y surgen algunos significados que ayudan a resolver parte de la actividad cotidiana, en particular la que se repite con cierta frecuencia, constituyendo un saber próximo de la conducta en la práctica del profesor.

\section{Conformación de imágenes}

Es el conjunto de interpretaciones que se configuran como conceptualizaciones teóricas y atienden a categorías externas que explican las concepciones del profesor. Estas imágenes son desarrolladas por el profesor durante el ejercicio de la profesión e involucran aspectos de la enseñanza y el aprendizaje tales como el aprendizaje de los alumnos, la metodología, la naturaleza de los contenidos, el papel de la programación y la evaluación, los fines y objetivos deseables. Tienen un fuerte poder orientador de la conducta profesional, a la vez que constituyen un saber autónomo y coherente con los saberes académicos del profesor. Se expresan más claramente en los momentos de programación, evaluación y, muy particularmente, en situaciones de diagnóstico de los problemas y conflictos que se dan en el aula.

Las imágenes escapan al propio control, tienden a bloquear los cambios en el dominio de lo concreto y favorecen cierta disociación entre el discurso pedagógico y la intervención docente. Es importante, en consecuencia, que los profesores se den cuenta de que, además de los conocimientos que han elaborado conscientemente, existen otros implícitos que 
deben intentar expresar para poder así comprender mejor una parte significativa de sus actuaciones ante los problemas de clase.

Las imágenes solo se pueden evidenciar con ayuda de otras personas que le brinden herramientas para analizar los modelos de enseñanza y de aprendizaje que se esconden en cada una de ellas. El carácter no explícito conlleva a bloquear los cambios en el dominio donde lo concretan y favorecen las disociaciones entre el discurso pedagógico y la práctica docente. Es entonces que la toma de conciencia de las imágenes representa parte del desarrollo profesional del profesor.

\section{Conformación de técnicas concretas}

Corresponden al conjunto de técnicas que establece el profesor a partir del conocimiento matemático y el conocimiento didáctico del contenido matemático que el profesor adquiere a medida que se involucra en los procesos de enseñanza y aprendizaje y profundiza en el conocimiento de los estándares.

Dichas técnicas están relacionadas directamente en el conocimiento de la formulación, organización y secuenciación del conocimiento escolar. En este componente de la experiencia, el profesor sabe plantear situaciones problemáticas en clase, elaborar tramas o mapas de contenidos en los que se integren las aportaciones de distintos tipos de conocimiento, elaborar y usar hipótesis sobre la posible progresión en la construcción del conocimiento con el análisis de los obstáculos que pueden aparecer en el proceso y con la determinación de diferentes niveles de formulación de los contenidos.

Las técnicas concretas implican el conocimiento de la existencia de concepciones de sus propios alumnos; reconocer que dichas concepciones se consideran como una variable en los procesos de enseñanza y aprendizaje, detectarlas e interpretarlas didácticamente y así emplear instrumentos de exploración adecuados en el contexto adecuado, teniendo presente que dichas concepciones cambian en un proceso de negociación y construcción compartida del conocimiento, para lo cual es vital la actuación del profesor.

La experiencia es entendida en esta investigación como un conocimiento que se adquiere más allá del ejercicio profesional a lo largo del tiempo, la cual involucra la reflexión y alimenta cada uno de los componentes descritos anteriores a través de un ciclo que comprende cinco fases:

Fase I. Comprensión, entendido como conocimiento comprensivo de la materia objeto de enseñanza y aprendizaje, en este caso matemáticas.

Fase II. Transformación del contenido enseñado a partir de las interpretaciones críticas, representaciones y adaptaciones en concordancia con las necesidades de aprendizaje de los estudiantes, las concepciones de ellos y las necesidades curriculares a quienes va dirigida la enseñanza. 
Fase III. Modificación en la instrucción, es decir, de la enseñanza propiamente dicha entendida como la actuación observable del docente en el aula. Se establecen rutinas, imágenes y técnicas acerca de la gestión del aula, la presentación de la información, la interacción con los alumnos, entre otros.

Fase IV. Reflexión, entendida como el proceso aprendizaje de la experiencia en la que se consolidan las rutinas, las imágenes y las técnicas. En esta fase el profesor evalúa su propia enseñanza, volviendo importante la revisión, la reconstrucción y el análisis crítico de la acción propia. Mediante la reflexión, el profesor ya está listo para alimentar el conocimiento de su experiencia.

Fase I. Nueva comprensión. Acá se cierra el ciclo, llevando de nuevo a la primera fase, el profesor ha enriquecido su comprensión de los propósitos de la instrucción, de la materia para su enseñanza, de sí mismo y de los alumnos, enriqueciendo su experiencia como tal.

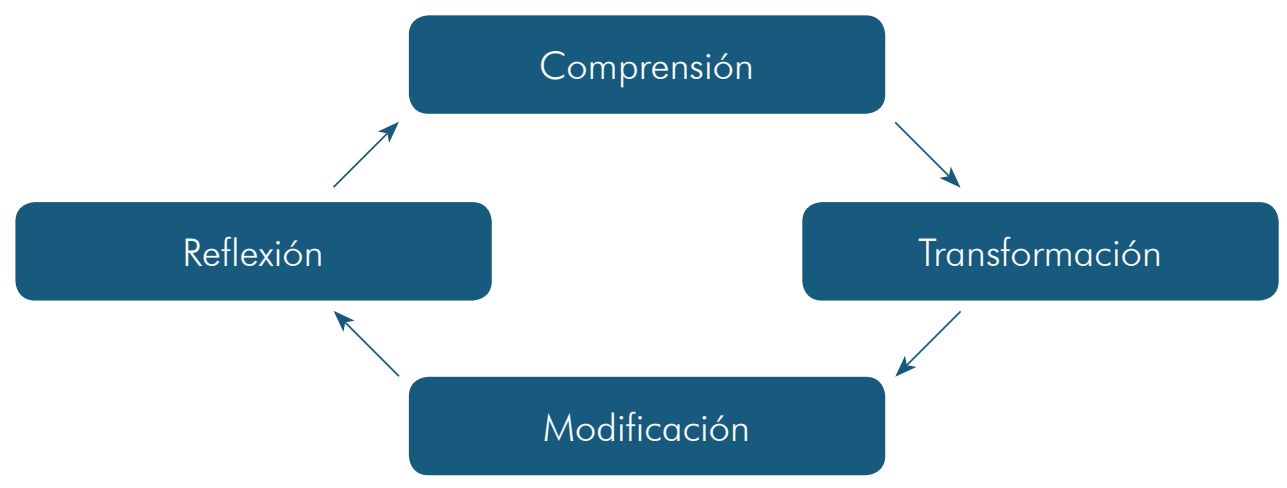

Figura 5. La experiencia

Fuente: elaboración propia

En la fase de transformación, en particular en la representación es menester el repertorio de representaciones de los contenidos que el docente dispone, volviendo importante el conocimiento didáctico del contenido matemático. En la misma fase, la adaptación necesita del conocimiento del profesor sobre las características generales del aprendizaje de los contenidos por parte de los alumnos y del manejo didáctico del contenido.

\section{Conclusiones}

El conocimiento especializado del profesor de matemáticas se organiza mediante la planificación y la ejecución de lo que él piensa, vive y lee. El docente al leer teorías, tener experien- cias, reflexionar sobre ellas y contrastarlas con su realidad va a lograr retroalimentar su propio conocimiento.

El conocimiento de la experiencia como componente del conocimiento especializado del profesor de matemáticas implica que dicho conocimiento sea para la práctica y no tanto desde la práctica; así, el énfasis se da en la comprensión y en la transformación que se dan a través de la reflexión. Esto elimina la falencia (no explícita) del modelo del conocimiento especializado del profesor de matemáticas presentado inicialmente, permitiendo comprender el proceso de desarrollo profesional aquí señalado, por lo que el conocimiento especializado del profesor de matemáticas debería ser representado de la manera que sigue: 


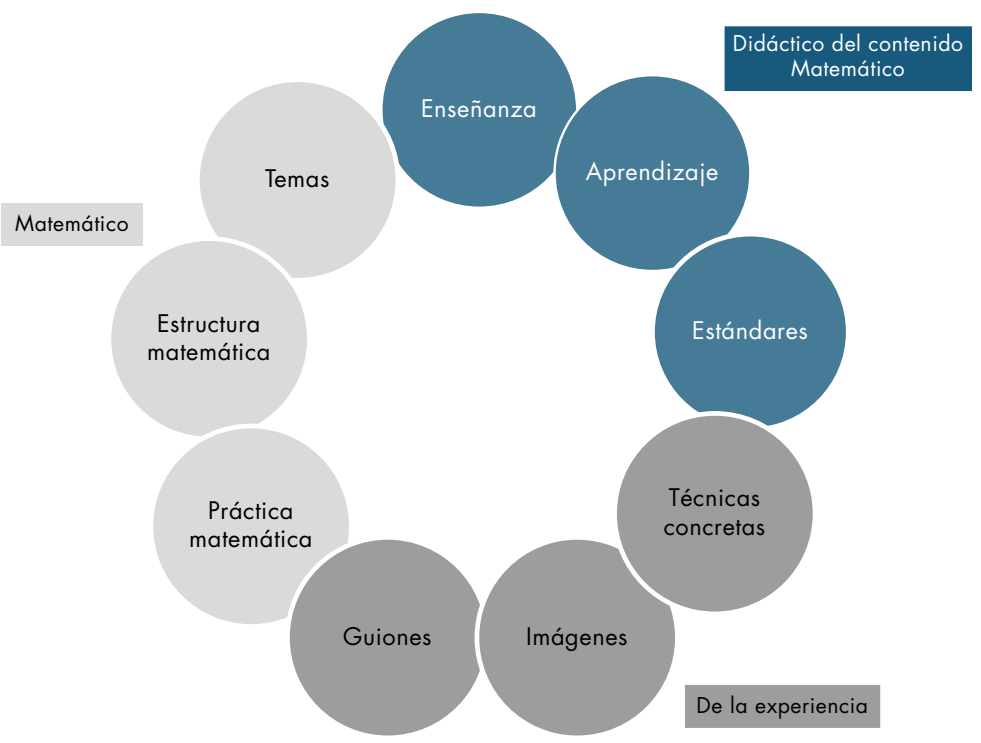

Figura 6. Componentes del conocimiento especializado del profesor de matemáticas Fuente: elaboración propia

\section{Referencias}

Askew, M., Brown, M., Rhodes, V., Johnson, D. y Wiliam, D. (1997). Effective teachers of numeracy. Londres: Kings College.

Ball, D. y Bass, H. (2009). With an eye on the mathematical horizon: knowingmathematics for teaching to learners' mathematical futures. Ahrestagung der Gesellschaft für Didaktik der Mathematik, USA: Michigan (49).

Ball, D. (1990). The mathematical understandings that prospective teacher bring to teacher education. Elementary School Journal (90), 449-466.

Ball, D. (2000). Bridging practices: Interwining content and pedagogy in teaching and learning to teach. Journal of Teacher Education, 51 (3), 241-247.

Ball, D., Thames, M. y Phelps, G. (2008). Content knowledge for teaching what makes it special. Journal of Teacher Education, 59(5), 389-407.

Bass, S., Didis, M., Erbas, A., Cetinkaya, B., Cakiroglu, E. y Alacaci, C. (2013). Teachers as investigators of students' written work: Does this approach provide an opportunity for profesional development. En B. Ubuz, C. Haser y M. Mariotti (1979), Critical Variables in Mathematics Education: Findings from a Survey of the Empirical Literature. Washington: NCTM, 1-10

Begle, E. (1979). Critical Variables in Mathematics Education: Findings from a Survey of the Empirical Literature. Washington: NCTM.

Climent, N. (2005). El desarrollo profesional del maestro de primaria respecto de la enseñanza de la matemática. Un estudio de caso. Michigan: Proquest Michigan University. 
Cooney, T. (1998). Conceptualizing the professional development of teachers. Selección de Conferencias del ICME 8. Sevilla.

Eisenberg, T. (1977). Begle revisited: Teacher knowledge and student achievement in algebra. Journal for Research in Mathematics Education, 8(3), 216-222.

Guacaneme, E. (2013). Conflictos para precisar el conocimiento disciplinar del profesor de matemáticas. En C. Flores, M. García, J. Hernández y L. Sosa (s. f.), Matemática Educativa: La formación de profesores. México: Universidad Autónoma de Guerrero.

Hill, H., Rowan. B. y Ball, D. (2005). Effects of teachers' mathematical knowledge for teaching on student achievement. American Educational Research Journal, 42(2), $371-406$.

Llinares, S. (2006). Aprendiendo a ver la enseñanza de la matemática. En S. Sbaragli y B. D’Amore (eds.) (s. f.), La Matemática e la seva Didattica, ventánni diimpegno, (pp. 177-180). Roma: Carocci Faber.

Muñoz Catalán, M.C., Contreras, L.C., Carrillo, J., Rojas, N., Montes, M.Á. y Climent, N. (2015). Conocimiento especializado del profesor de matemáticas (MTSK): un modelo analítico para el estudio del conocimiento del profesor de matemáticas. La Gaceta de la Real Sociedad Matemática Española, 18 (3), 1801-1817.

Porlán, R. y Rivero, A. (1998). El conocimiento de los profesores. Una propuesta formativa en el área deficiencias. Sevilla: Diada Editora.

Schifter, D. y Simon, M. (1992). Assessing teacher's development of a constructivist view of mathematics learning. Teaching and Teacher education, 8(2), 187-197.

Schwab, J. (1978). Education and the structure of the disciplines. In I. Westbury \& N. Wilkof (Eds.), Science, curriculum, and liberal education, (pp. 229-272). Chicago: University of Chicago Press.

Shulman, L. (1986). Those who understand: Knowledge growth in teaching. Educational Researcher, 15(2), 4-14.

Shulman, L. (1987). Knowledge and teaching: Foundations of the new reform. Harvard Educational Review, 57(1), 1-23.

Shulman, L. y Grossman, P. (1988). Knowledge growth in teaching: A final report to the Spencer foundation. Stanford: Stanford University

\section{Para citar este artículo}

Lima, I. (2017). Perspectivas del conocimiento especializado del profesor de matemáticas como elemento de su desarrollo profesional. Tecné, Episteme y Didaxis, TED, 42, 175-191. 